\title{
Repeatability and internal consistency of abdominal 2D and 4D PC MR flow measurements
}

\author{
Andrew Wentland ${ }^{1,2^{*}}$, Thomas M Grist ${ }^{1,2}$, Oliver Wieben ${ }^{1}$ \\ From 15th Annual SCMR Scientific Sessions \\ Orlando, FL, USA. 2-5 February 2012
}

\begin{abstract}
Background
We have recently demonstrated the benefits of a radially undersampled 4D-MR flow acquisition (PC-VIPR) $[1,2]$ for angiographic imaging of the renal vasculature in humans [3] and for transstenotic pressure gradients in a swine model [4]. Validation of velocity measurements in vivo with non-MRI methods is desirable, but not possible. The purpose of this study was to assess the repeatability of 2D and 4D-PC flow measurements in humans and to assess the internal consistency of arterial in-flow and out-flow measurements in the renal vasculature.
\end{abstract}

\section{Methods}

Eight healthy volunteers (mean age $=26.9 \pm 2.5$ years) were scanned on a 3TMR scanner with a 32-channel phasedarray torso coil. Subjects refrained from eating for a minimum of four hours prior to the MR examination. 2DPC scan planes were prescribed in the supra- and infrarenal aorta and in each renal artery (Fig.1) with a double-oblique orientation. A radially undersampled 4D-PC technique (PC-VIPR) with large volume coverage over the abdomen and 3-directional velocity encoding was also acquired. Each subject then got off of the scanner bed for five minutes and subsequently returned to repeat the MR protocol.

Total flow over the cardiac cycle was measured with in-house-developed software. To test for internal consistency, differences were computed between the suprarenal aortic flow $\left(Q_{\text {in }}\right)$ and the sum of flow measurements in the renal arteries and infrarenal aorta $\left(\mathrm{Q}_{\text {out }}\right)$; differences were normalized by the average of $\mathrm{Q}_{\text {in }}$ and $\mathrm{Q}_{\text {out }}$. 2D and 4D percent differences were compared with a Student's t-test. The repeatability of flow measurements was assessed with Pearson correlation and Bland-Altman analysis.

${ }^{1}$ Medical Physics, UW - Madison, Madison, WI, USA

Full list of author information is available at the end of the article

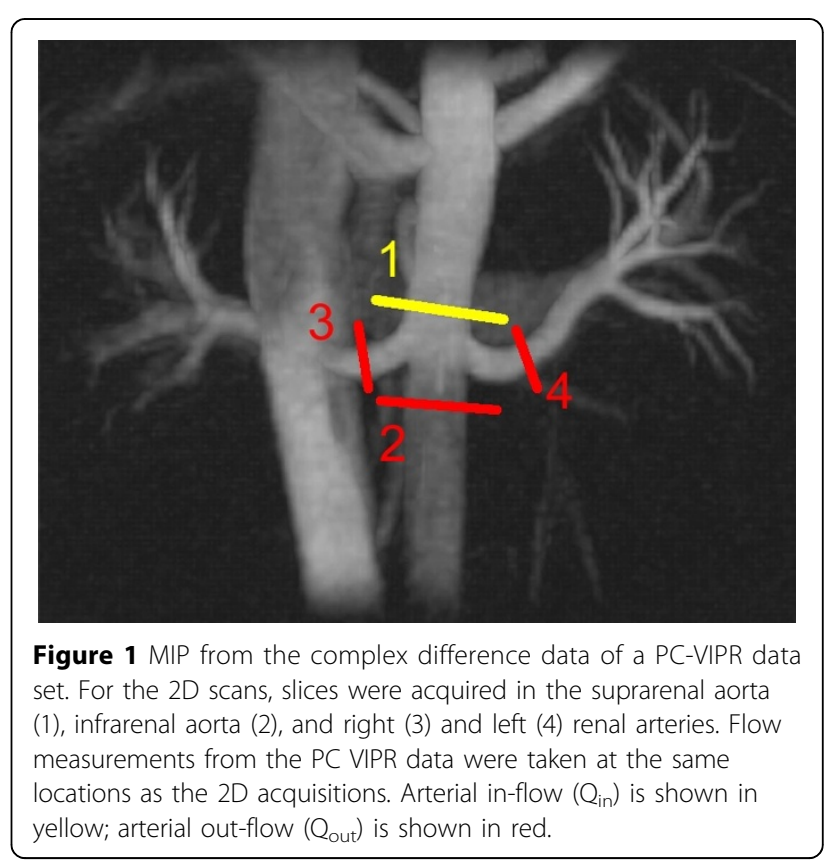

\section{Results}

For all 16 sets of 2D measurements, average $\mathrm{Q}_{\text {in }}(2404.8$ $\pm 632.5 \mathrm{ml} / \mathrm{min})$ was similar to average $\mathrm{Q}_{\text {out }}(2259.1$ $\pm 449.4 \mathrm{ml} / \mathrm{min}$ ). Similarly, for all 16 sets of $4 \mathrm{D}$ measurements, average $\mathrm{Q}_{\text {in }}(1517.5 \pm 585.2 \mathrm{ml} / \mathrm{min})$ was similar to average $Q_{\text {out }}(1343.9 \pm 410.8 \mathrm{ml} / \mathrm{min})$. 4D flow measurements tended to be lower in magnitude than 2D flow measurements. Normalized, the total flow difference for all $162 \mathrm{D}$ sets of measurements $(18.0 \pm 16.5 \%)$ was greater than the mean percent difference of all $164 \mathrm{D}$ measurements $(12.4 \pm 8.8 \%$; $=0.053$;Fig.2A). The repeatability of both $2 \mathrm{D}\left(\mathrm{r}=0.91, \mathrm{p}<1 \times 10^{-12}\right)$ and $4 \mathrm{D}\left(\mathrm{r}=0.86, \mathrm{p}<1 \times 10^{-9}\right)$ flow measurements was strongly correlative. Bland-Altman analysis demonstrated small overall flow differences 

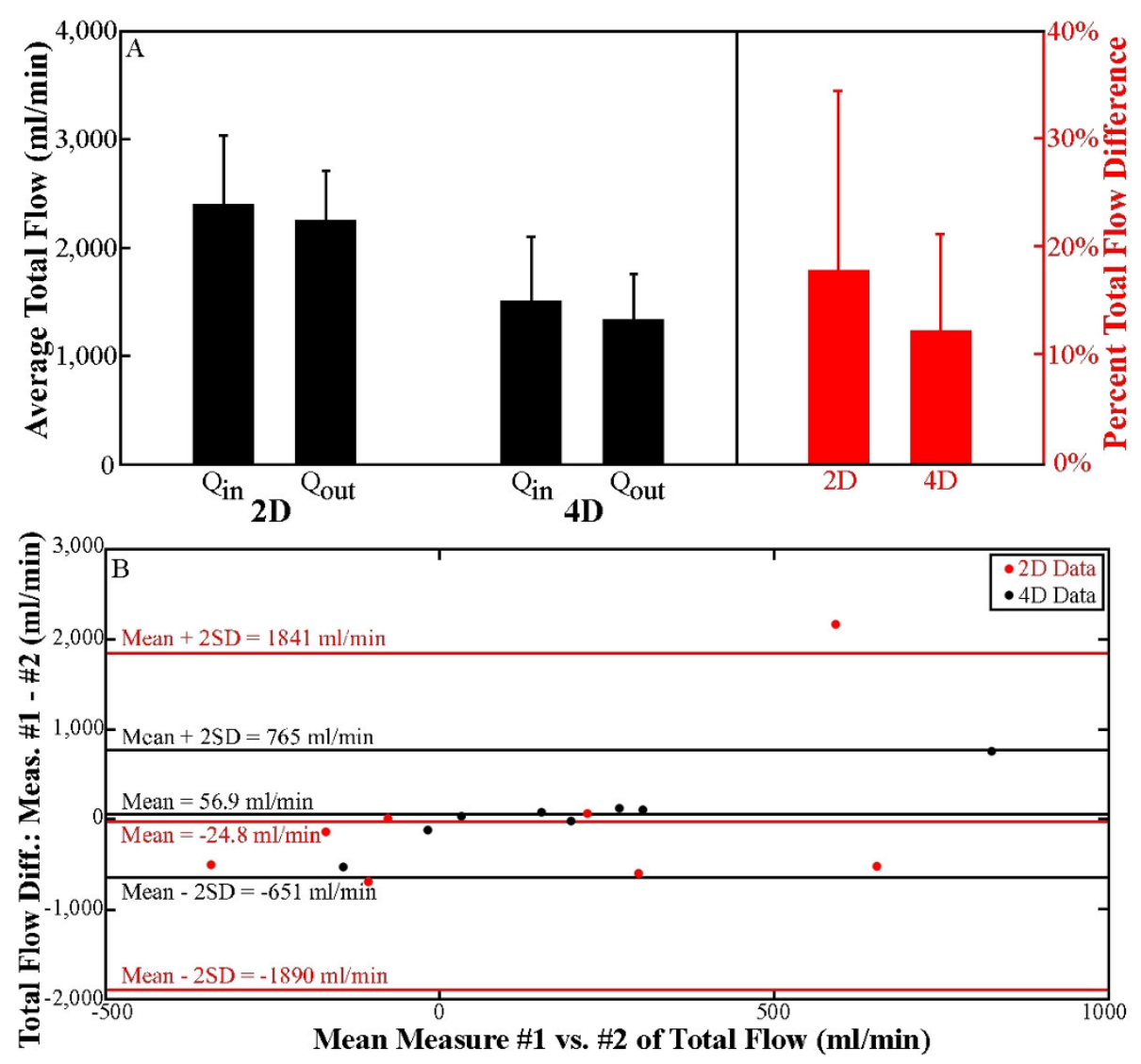

Figure 2 A. Average absolute and percent total flow differences for $2 D$ and $4 D P C$ flow measurements. Flow differences were computed as the difference between suprarenal aortic flow $\left(\mathrm{Q}_{\mathrm{in}}\right)$ and the sum of the flow in the infrarenal aorta and two renal arteries ( $\left.\mathrm{Q}_{\text {out }}\right)$. Measurements were normalized by the average of these two values. Bars are standard deviation. B. Bland-Altman plots for the repeatability of 2D and 4D flow difference measurements.

for both the $2 \mathrm{D}($ mean $\pm 2 \mathrm{SD}=-24.8 \pm 1865 \mathrm{ml} / \mathrm{min})$ and $4 \mathrm{D}$ $(\mathrm{mean} \pm 2 \mathrm{SD}=56.9 \pm 708 \mathrm{ml} / \mathrm{min})$ techniques (Fig.2B).

\section{Conclusions}

Flow measurements in healthy volunteers revealed that 4D measurements tended to be more internally consistent than 2D measurements, with average differences slightly greater than $10 \%$. The repeatability of the $2 \mathrm{D}$ and 4D data were similar. These results are favorable compared to a previous report [5] comparing 2D and 4D PC flow measurements, despite the compounding error of multiple flow measurements for this check on internal consistency.

\section{Author details}

${ }^{1}$ Medical Physics, UW - Madison, Madison, WI, USA. ²Radiology, UW -

Madison, Madison, WI, USA.

Published: 1 February 2012

\section{References}

1. AJNR. 26:746
2. MRM. 60:1329.

3. Radiology. 258:254.

4. Radiology. 261:266

5. MRM. 60:1218.

doi:10.1186/1532-429X-14-S1-W13

Cite this article as: Wentland et al:: Repeatability and internal consistency of abdominal 2D and 4D PC MR flow measurements. Journal of Cardiovascular Magnetic Resonance 2012 14(Suppl 1):W13.

Submit your next manuscript to BioMed Central and take full advantage of:

- Convenient online submission

- Thorough peer review

- No space constraints or color figure charges

- Immediate publication on acceptance

- Inclusion in PubMed, CAS, Scopus and Google Scholar

- Research which is freely available for redistribution 